\title{
The Role of Sialic Acid-Binding Receptors (Siglecs) in the Immunomodulatory Effects of Trypanosoma cruzi Sialoglycoproteins on the Protective Immunity of the Host
}

\author{
Alexandre Morrot \\ Institute of Microbiology, Federal University of Rio de Janeiro, CCS, Sala D1-035, Avenida Carlos Chagas Filho 373, \\ Cidade Universitária, Ilha do Fundão, 21.941-902 Rio de Janeiro, RJ, Brazil \\ Correspondence should be addressed to Alexandre Morrot; morrot@micro.ufrj.br
}

Received 17 November 2013; Accepted 10 December 2013

Academic Editors: M. Salio, A. R. Satoskar, R. Teasdale, and A. G. Zapata

Copyright (C) 2013 Alexandre Morrot. This is an open access article distributed under the Creative Commons Attribution License, which permits unrestricted use, distribution, and reproduction in any medium, provided the original work is properly cited.

Chagas disease is caused by the protozoan parasite Trypanosoma cruzi and is an important endemic infection in Latin America. Lately, it has also become a health concern in the United States and Europe. Most of the immunomodulatory mechanisms associated with this parasitic infection have been attributed to mucin-like molecules on the T. cruzi surface. Mucins are high molecular weight glycoproteins that are involved in regulating diverse cellular activities in both normal and pathological conditions. In Trypanosoma cruzi infection, the parasite-derived mucins are the main acceptors of sialic acid and it has been suggested that they play a role in various host-parasite interactions during the course of Chagas disease. Recently, we have presented evidence that sialylation of the mucins is required for the inhibitory effects on $\mathrm{CD}^{+} \mathrm{T}$ cells. In what follows we propose that signaling via sialic acid-binding Ig-like lectin receptors for these highly sialylated structures on host cells contributes to the arrest of cell cycle progression in the G1 phase and may allow the parasite to modulate the immune system of the host.

\section{Trypanosoma cruzi Infection and the Immunopathology of Chagas Disease}

Chagas' disease or American trypanosomiasis is a tropical parasitic illness affecting nearly 20 million people in the Americas $[1,2]$. The disease is caused by the protozoan flagellated parasite Trypanosoma cruzi, transmitted to humans by haematophagous insects known as triatomines (Reduviidae family). The complex life cycle of T. cruzi includes epimastigote and metacyclic trypomastigote stages in the insect vector and bloodstream trypomastigote and intracellular amastigotes in the vertebrate host [3]. In the latter, the Trypanosoma cruzi infects several cell types, including monocytes, fibroblasts, endothelial cells, and muscle cells [4-9]. This capacity to invade a wide range of host cells is associated with increased tissue inflammation and evokes a strong immunological response. This host protective response results from host tissue damage due to increased infiltration of leukocytes to the inflammatory sites, producing proinflammatory mediators, including cytokines, chemokines, and nitric oxide, among other factors [10-14]. Approximately $30 \%$ of infected patients develop symptoms of the disease in their lifetime; these include cardiomyopathy, neuropathies, and dilatation of the colon or esophagus [15].

The pathogenesis of Chagas disease is controversial and distinct hypotheses have been considered, including autoimmune manifestations and parasite-driven tissue damage [1618 . Whatever is the case, it is accepted that the events occurring during the acute phase of $T$. cruzi infection determine the pathological features that arise later during the chronic phase of the disease [19]. The initial stages of the infection are characterized by the induction of nonspecific lymphoproliferation [20]. This phenomenon involves extensive polyclonal activation of lymphocytes. There is an increased frequency of immunoglobulin-secreting B cells with the typical isotype profile for IgG2a and IgG2b in peripheral lymphoid organs, and the majority of these polyclonal activated B cells secrete nonspecific antibodies with low affinity for T. cruzi antigens. The $\mathrm{T}$ cells are also polyclonally expanded in the course of infection, but it seems that the massive polyclonal activation targets the minor CD5B and $\gamma \delta$ T lymphocyte subsets [21]. 
This polyclonal activation is believed to have a role in inducing autoimmune reactions during Chagas disease. There are numerous reports of T. cruzi antigens cross-reactive with heart and neural tissues [22-24], but these autoantibodies or autoreactive $\mathrm{T}$ cells are believed to play secondary roles in the pathogenesis of Chagas disease as the affinity of the peripheral lymphocyte repertoire with cross-reactive antigens is low due to the negative selection that they undergo during the process of central tolerance [25-27]. However, it seems that the polyclonal activation in Chagas disease has a role in the immunosuppressive mechanisms associated with Trypanosoma cruzi infection. As the activation and survival of lymphocytes are determined by competitive access to niches containing antigen and cytokines in lymphoid tissues, it is possible that the polyclonal activation of lymphocytes dampens the protective immune response by limiting the competitiveness of antigen-specific lymphocyte relative to the high frequency of polyclonally expanded T/B cells [28-30]. These events could account for the immunosuppression seen in both mice and humans in the acute phase of Chagas disease $[8,31-39]$. In addition to these alterations in peripheral lymphoid organs, the thymus is also a target for parasite-induced changes of the host immune system. During the acute phase of the disease, severe thymic atrophy occurs, mainly due to apoptotic depletion of $\mathrm{CD} 4^{+} \mathrm{CD}^{+}$double-positive (DP) thymocytes in the cortical area of the thymic lobules [40].

In spite of this depletion in the thymus, there is also an abnormal release of DP cells into the periphery, resulting in a more than 15 -fold increase in DP cell numbers in subcutaneous lymph nodes. This premature thymic emigration of immature thymocytes is likely to be a result of alterations of the thymic microenvironment, with enhanced deposition of cell migration-related molecules such extracellular matrix (ECM) proteins and chemokines CXCL12 and CCL21, which can influence the migration of developing thymocytes during thymopoiesis [41-44]. Interestingly, we have shown that, in contrast to physiological conditions, the DP cells released into the periphery during the course of the infection acquire an activated phenotype similar to that described for activated single-positive T cells. Furthermore, we showed that the presence of activated DP cells in the periphery is correlated with the development of the severe clinical form of chronic human Chagas disease [40].

Despite the changes observed in the thymus during infection, we have shown that the intrathymic expression of the autoimmune regulator factor (Aire) and tissue-restricted antigen (TRA) genes is normal. In addition, expression of the proapoptotic Bim protein in thymocytes is unchanged, showing that the thymic atrophy has no effect on the checkpoints required for clonal T cell deletion. In a chicken egg ovalbumin- (OVA-) specific T cell receptor (TCR) transgenic system, the administration of OVA peptide to infected mice undergoing thymic atrophy promoted OVA-specific thymocyte apoptosis, further indicating that the negative selection process is normal during infection [40]. These findings indicate that the key intrathymic elements necessary for negative selection of thymocytes undergoing maturation during thymopoiesis remain functional during the acute chagasic thymic atrophy.
However, the fact that negative selection still operates in the thymus [40], which is also a locus of colonization by T. cruzi [45], may lead to newly generated T cells tolerant to the invading pathogen. During thymic colonization, the pathogen could be able to target the thymic DCs to promote clonal deletion of recycling activated pathogen-specific $\mathrm{T}$ cells that migrate from the thymus to clear the infection [46]. Although we showed that the key intrathymic elements responsible for negative selection of thymocytes are active during thymopoiesis [40], deletion of activated-recycling T cells specific for T. cruzi parasites in the thymus could play a role in the central tolerance mechanism promoting pathogen persistence.

\section{The Immunosuppressive Effects of Trypanosoma cruzi-Derived Mucins}

As in any chronic infectious disease, the Trypanosoma cruzi parasite has evolved the capacity to survive in its vertebrate host by weakening the host's immune response [35, 47-50]. In both humans and experimental models of T. cruzi infection, the acute phase of Chagas disease is marked by a state of immunosuppression [31-34, 36, 38]. This subversion of the host protective immune response at the beginning of infection in the acute phase of T. cruzi infection is responsible for the persistence of the parasite and the establishment of a chronic disease [51-53]. T. cruzi in fact provides a good example of such a strategy. T cells from infected mice show reduced IL-2 expression and low proliferative responses to mitogens $[32,33,36]$. In addition, $\mathrm{CD} 4^{+} \mathrm{T}$ cells from infected mice when activated by stimulation of the $\mathrm{T}$ cell receptor show enhanced apoptosis increasing the unresponsiveness of host immunity. These characteristics are indicative of an immunosuppressed state. This immunodeficiency is also characterized by reduced protective humoral responses [54-56].

The host-parasite interplay underlying the immunosuppression during Chagas disease has been elucidated. Independent studies have demonstrated that T. cruzi membrane glycoproteins are critical for damping host protective immunity. The parasite surface is covered by sialic acid residues which are transferred from host glycoconjugates to the terminal $\beta$ galactosyl residues of mucin-like molecules on its surface by a unique enzyme, the trans-sialidase [57-60] (Figure 1). These T. cruzi mucins are the most abundant glycoproteins on the surface of the parasite and consist of O-glycosylated Thr/Ser/Pro-rich proteins. The T. cruzi mucin-like molecules consist of a large repertoire of glycoproteins encoded by more than 800 genes comprising approximately $1 \%$ of the parasite genome [61-63]. These molecules play a key role in the invasion of the host and subversion of its immune system. It has been shown, for instance, that the sialylated mucins mask parasite antigenic determinants, thus protecting the parasite from host attack by anti-galactosyl antibodies and complement factor B [64-67].

The sialylated glycoconjugates have been shown to modulate the host dendritic cell function by suppressing the production of the proinflammatory cytokine IL-12, as well as T cell activation and proliferation in response to mitogens and antigens $[68,69]$ (Figure 1). These effects may involve action 


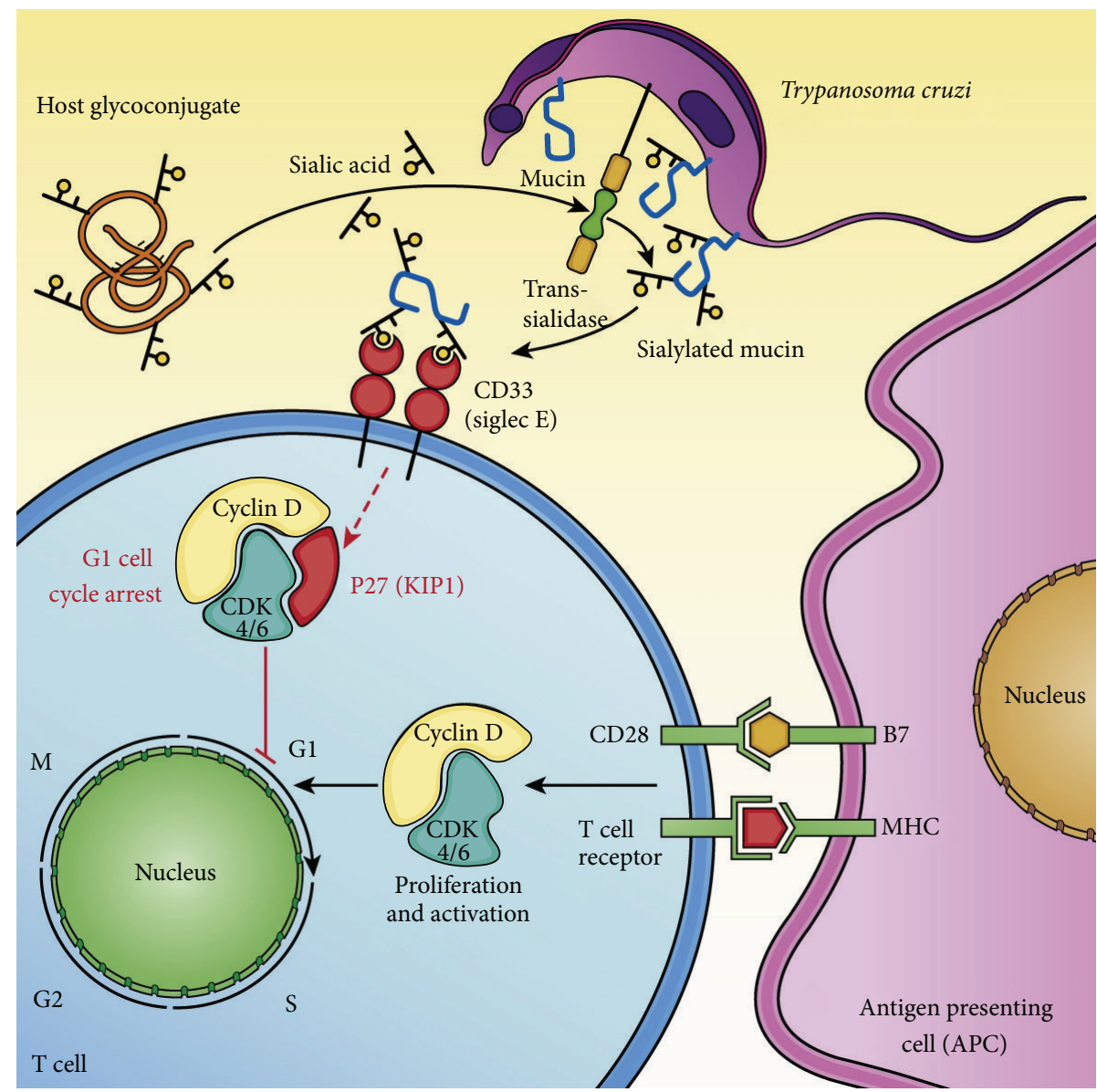

FIGURE 1: Model depicting the inhibitory effect of Trypanosoma cruzi sialoglycoproteins on T cell activation. Schematic diagram showing the sialylation of O-linked oligosaccharides of mucin-derived trypomastigotes mediated by the surface-associated T. cruzi parasite trans-sialidase. The parasite trans-sialidase, which can also be shed into the bloodstream or tissue interstitium after cleavage of its glycosylphosphatidylinositol (GPI) anchor by the action of a phosphatidylinositol-phospholipase C, transfers sialic-acid residues from host glycoconjugates to parasite mucins. It has been demonstrated in other studies that the T. cruzi parasite-derived mucins bind to mammalian host cell receptors such as the acid-binding Ig-like lectin receptor Siglec-E (CD33) and undermine host defence mechanisms. In CD4 ${ }^{+} \mathrm{T}$ cells, we showed that the Siglec-E receptor inhibits the mitogenic responses upon $\mathrm{T}$ cell receptor stimulation. The initiation of the G1 to $\mathrm{S}$ transition during antigenic/mitogenic T cell expansion is mediated by cyclin D and cyclin-dependent kinases CDK2 or CDK6, which are induced and together initiate the G1/S transition. We have shown that the G1/S transition is significantly inhibited by the sialyl terminal residues of T. cruzi mucins and we propose that this phenomenon is mediated by its interaction with the Siglec-E receptor. The interaction of CD4 ${ }^{+} \mathrm{T}$ cells with the sialylated form of the parasite mucin leads to induction of $\mathrm{p} 27 / \mathrm{Kipl}$, a member of the family of CDK inhibitors that negatively regulate the G1 to $S$ transition, so damping $\mathrm{T}$ cell-mediated immune responses by inducing $\mathrm{T}$ cell cycle arrest.

at the transcriptional level, since the T. cruzi mucins inhibit transcription of IL-2 gene [32, 33]. Moreover, other studies have shown that these sialoglycoproteins also inhibit early events in T cell activation such as tyrosine phosphorylation of the adapter protein SLP-76 and the tyrosine kinase ZAP70 [36]. The inhibitory effects of the T. cruzi mucins were recently examined in vivo. We found that exposure of mice to exogenous T. cruzi-derived mucins during infection with Trypanosoma cruzi increased their susceptibility to infection and led to increased parasitemia and heart damage. These alterations were correlated with a lower frequency of IFN$\gamma$-producing $\mathrm{CD}^{+}$and $\mathrm{CD}^{+} \mathrm{T}$ cell responses, in addition to decreased levels of both splenic IFN- $\gamma$ and TNF- $\alpha$ cytokines [69]. These data indicated that the parasite mucins influence the course of the parasite-host interaction during the acquisition of cell-mediated adaptive immune responses, damping protective host responses in order to establish persistent chronic infections.

\section{The Sialic Motifs of Trypanosoma cruzi Mucins Target Host Sialic Acid-Binding Receptors (Siglecs) during Parasite Immunomodulation}

Sialic acids are found on all cell surfaces of a variety of organisms, including pathogens that interact with vertebrates [70]. In Trypanosoma cruzi infections, the sialylated glycoconjugates play important roles in the initiation, persistence, and pathogenesis of Chagas' disease [64-67, 71]. Their target 
receptors in the host have only been identified recently. There is evidence that sialylated Tc Muc can interact with Siglec-E (CD33) (Figure 1), a member of the Siglec family of sialic acidbinding Ig-like lectins found mainly on cells of the immune system $[72,73]$. The Siglec receptors are structures similar to lectins and have variable specificity for sialic acid-containing ligands [74]. Siglecs have immunoreceptor tyrosine-based inhibitory motifs (ITIMs) in their cytosolic tails, which suggests that they are able to perform inhibitory functions when they bind sialylated carbohydrates. In fact, many Siglecs are inhibitory receptors that mediate a variety of different inhibitory functions in the immune system, such as regulation of the inflammation mediated by damage-associated and pathogen-associated molecular patterns (DAMPs and PAMPs), promoting the tolerance of $\mathrm{B}$ lymphocytes and modulating the activation of dendritic cells and the activation of $\mathrm{T}$ cells $[70,74]$.

The receptor Siglec-E target of the T. cruzi mucins is a restricted leukocyte antigen mainly expressed on mouse phagocytic cells and on antigen-presenting cells (APCs) including macrophages and dendritic cells $[75,76]$. Binding of $T$. cruzi to Siglec-E-expressing cells is followed by rapid mobilization of Siglec-E into the contact zone between parasite and host cells. This triggering of Siglec-E modulates the activity of dendritic cells, leading to lower production of IL-12, which is important for Th1 responses $[72,73]$. In addition, triggering of Siglec-E on dendritic cell surfaces with cross-linking antibodies reduces the capacity of $\mathrm{T}$ cells to be activated and proliferate [72]. This phenomenon may contribute to the parasiteinduced modulation of host immunity by damping $\mathrm{T}$ cell protective responses.

The capacity of T. cruzi mucins to modulate the adaptive immune response does not seem to be restricted to T. cruzi. The inefficient host immune response to cancer antigens is at least in part due to the presence of carcinoma-associated mucins [77-81]. However, the mechanisms involved in these effects of mucin-like molecules on the immune system are not well understood. In this connection, we have recently shown that the T. cruzi mucin is able to inhibit $\mathrm{CD} 4^{+} \mathrm{T}$ cell proliferation by inducing $\mathrm{T}$ cell anergy. We showed that exposure of $\mathrm{CD}^{+} \mathrm{T}$ cells to parasite mucins significantly reduced IL2 secretion in response to TCR activation [69]. Furthermore, our findings indicate that the state of anergy induced in the $\mathrm{T}$ cells by the parasite mucins is not reversed by exogenous IL-2, implying that the IL-2 pathway is irreversibly impaired upon activation of $\mathrm{CD} 4^{+} \mathrm{T}$ cells in the presence of T. cruzi mucins. These inhibitory effects of T. cruzi mucins also extend to the other aspects of $\mathrm{CD} 4^{+} \mathrm{T}$ cell differentiation pathways, as we have shown that the parasite mucin inhibits the production of cytokines during TCR stimulation, including those known to protect against parasite infections, such as IFN- $\gamma$ and TNF- $\alpha$ [69].

We have also asked whether the sialylation of the $T$. cruzi mucin influences the strength of its inhibition of $\mathrm{CD}^{+} \mathrm{T}$ cells. We found that the removal of the sialic acid terminal residues by neuraminidase treatment partially abolished the inhibitory effects of the mucin on $\mathrm{CD}^{+} \mathrm{T}$ cell proliferative responses, indicating a possible role for the sialic acid-binding Ig-like lectin receptors expressed by $\mathrm{T}$ cells in the inhibitory effects of the parasite mucins [69]. In fact, we showed for the first time that triggering of CD33 on $\mathrm{CD}^{+}{ }^{+} \mathrm{T}$ cells with anti-Siglec $\mathrm{E}$ antibodies significantly inhibited the proliferation of stimulated $\mathrm{T}$ cells. We therefore propose that the $\mathrm{T}$ cell surface mucin receptor Siglec-E is implicated in the inhibition of $\mathrm{T}$ cell proliferation [69] (Figure 1).

When dissecting the signaling pathway of the T. cruzimediated inhibition of $\mathrm{T}$ cell responses, we found that the parasite mucin was able to induce G1 cell cycle arrest associated with upregulation of the cyclin D inhibitor p27(kip1) and downmodulation of cyclin D3 on activated $\mathrm{CD}^{+}{ }^{+} \mathrm{T}$ cells [69]. p27 is a phosphatase regulator that participates in the G1 cell cycle arrest checkpoint [82-84]. In contrast, when $\mathrm{CD} 4^{+}$ $\mathrm{T}$ cells were polyclonally activated in the presence of desialylated T. cruzi mucin the signaling profile was reversed as demonstrated by the upregulation of cyclin D3 and downmodulation of $\mathrm{p} 27$ (kip1), a profile similar to that described for control TCR-activated T cells. These data indicate that T. cruzi mucins exert antiproliferative effects on $\mathrm{CD}^{+}{ }^{+} \mathrm{T}$ cells, inducing G1 phase arrest by increasing the amount of p27(kipl), an immune modulatory effect that is potentiated by the sialic acid terminal residues of the parasite mucins [69] (Figure 1).

\section{Concluding Remarks}

Several independent studies have provided evidence that $T$. cruzi mucins are involved in $\mathrm{T}$ cell responses by affecting the activation, differentiation, and expansion of $\mathrm{T}$ cells. Our results indicate that Tc Muc mediates the inhibitory effects on $\mathrm{CD} 4^{+} \mathrm{T}$ expansion and cytokine production by blocking cell cycle progression in the G1 phase. We propose that the sialyl motif of Tc Muc is able to interact with sialic acidbinding Ig-like lectins (Siglecs) on $\mathrm{CD}^{+} \mathrm{T}$ cells, which may allow the parasite to modulate the immune system. It is likely that Siglec-E is involved in this effect. It is noteworthy that our studies revealed that the mucin derived from $T$. cruzi parasites upregulates the expression of the mitogen inhibitor p27(kip1) associated with the G1-phase cell cycle arrest, and this phenomenon is potentiated by the sialyl terminal residues of $T$. cruzi mucins which are important for its inhibitory effects on $\mathrm{T}$ cells. These findings point to an important feature of pathogen virulence as we show that $T$. cruzi modifies the cell cycle phase of $\mathrm{T}$ cells to make them anergic to antigenic stimulation during the acquisition of protective immunity. Actually other diseases such as virus infections and cancers modulate the cell cycle to serve their own purposes $[85,86]$. For instance, expression of cell cycle proteins like cyclins or cyclin-dependent kinases can be modulated in order to interrupt the cell cycle at a phase that is advantageous for a virus to replicate [86]. Understanding these intricate effects on cell cycle checkpoints, which play a role in damping natural and acquire immunity to invading microorganisms, will help us to elucidate the interplay between the virulence factors of infectious pathogens and the host immune response to infection in illnesses such as Chagas disease. 


\section{Acknowledgments}

This work was supported by grants from Conselho Nacional de Desenvolvimento Científico e Tecnológico do Brasil (CNPq), Fundação de Amparo à Pesquisa do Estado do Rio de Janeiro (FAPERJ), and Fundação Oswaldo Cruz.

\section{References}

[1] A. L. Ribeiro, M. P. Nunes, M. M. Teixeira, and M. O. Rocha, "Diagnosis and management of Chagas disease and cardiomyopathy," Nature Reviews Cardiology, vol. 9, pp. 576-589, 2012.

[2] M. A. Barry, J. E. Weatherhead, P. J. Hotez, and L. Woc-Colburn, "Childhood parasitic infections endemic to the United States," Pediatric Clinics of North America, vol. 60, pp. 471-485, 2013.

[3] E. S. Garcia, F. A. Genta, P. de Azambuja, and G. A. Schaub, "Interactions between intestinal compounds of triatomines and Trypanosoma cruzi," Trends in Parasitology, vol. 26, no. 10, pp. 499-505, 2010.

[4] E. Ortega-Barria and M. E. Pereira, "Entry of Trypanosoma cruzi into eukaryotic cells," Infectious Agents and Disease, vol. 1, no. 3, pp. 136-145, 1992.

[5] B. A. Burleigh and A. M. Woolsey, "Cell signalling and Trypanosoma cruzi invasion," Cellular Microbiology, vol. 4, no. 11, pp. 701-711, 2002.

[6] W. de Souza, T. M. de Carvalho, and E. S. Barrias, "Review on Trypanosoma cruzi: host cell interaction," International Journal of Cell Biology, vol. 2010, Article ID 295394, 18 pages, 2010.

[7] M. C. Fernandes and N. W. Andrews, "Host cell invasion by Trypanosoma cruzi: a unique strategy that promotes persistence," FEMS Microbiology Reviews, vol. 36, no. 3, pp. 734-747, 2012.

[8] F. Nagajyothi, F. S. Machado, B. A. Burleigh et al., "Mechanisms of Trypanosoma cruzi persistence in Chagas disease," Cellular Microbiology, vol. 14, no. 5, pp. 634-643, 2012.

[9] F. Y. Maeda, C. Cortez, and N. Yoshida, "Cell signaling during Trypanosoma cruzi invasion," Frontiers in Immunology, vol. 3, article 361, 2012.

[10] H. B. Tanowitz, J. P. Gumprecht, D. Spurr et al., "Cytokine gene expression of endothelial cells infected with Trypanosoma cruzi," Journal of Infectious Diseases, vol. 166, no. 3, pp. 598-603, 1992.

[11] H. Huang, T. M. Calderon, J. W. Berman et al., "Infection of endothelial cells with Trypanosoma cruzi activates NF- $\kappa \mathrm{B}$ and induces vascular adhesion molecule expression," Infection and Immunity, vol. 67, no. 10, pp. 5434-5440, 1999.

[12] B. Chandrasekar, P. C. Melby, D. A. Troyer, and G. L. Freeman, "Differential regulation of nitric oxide synthase isoforms in experimental acute Chagasic cardiomyopathy," Clinical and Experimental Immunology, vol. 121, no. 1, pp. 112-119, 2000.

[13] M. M. Teixeira, R. T. Gazzinelli, and J. S. Silva, "Chemokines, inflammation and Trypanosoma cruzi infection," Trends in Parasitology, vol. 18, no. 6, pp. 262-265, 2002.

[14] S. Mukherjee, H. Huang, S. B. Petkova et al., "Trypanosoma cruizi infection activates extracellular signal-regulated kinase in cultured endothelial and smooth muscle cells," Infection and Immunity, vol. 72, no. 9, pp. 5274-5282, 2004.

[15] A. Prata, "Clinical and epidemiological aspects of Chagas disease," The Lancet Infectious Diseases, vol. 1, no. 2, pp. 92-100, 2001.

[16] N. Gironès, H. Cuervo, and M. Fresno, "Trypanosoma cruziinduced molecular mimicry and Chagas' disease," Current
Topics in Microbiology and Immunology, vol. 296, pp. 89-123, 2005.

[17] F. R. Gutierrez, P. M. Guedes, R. T. Gazzinelli, and J. S. Silva, "The role of parasite persistence in pathogenesis of Chagas heart disease," Parasite Immunology, vol. 31, no. 11, pp. 673-685, 2009.

[18] E. Cunha-Neto, P. C. Teixeira, L. G. Nogueira, and J. Kalil, "Autoimmunity," Advances in Parasitology, vol. 76, pp. 129-152, 2011.

[19] C. R. Marinho, M. R. D’Império Lima, M. G. Grisotto, and J. M. Alvarez, "Influence of acute-phase parasite load on pathology, parasitism, and activation of the immune system at the late chronic phase of Chagas' disease," Infection and Immunity, vol. 67, no. 1, pp. 308-318, 1999.

[20] P. Minoprio, S. Itohara, C. Heusser, S. Tonegawa, and A. Coutinho, "Immunobiology of murine T. Cruzi infection: the predominance of parasite-nonspecific responses and the activation of TCRI T cells," Immunological Reviews, no. 112, pp. 183-207, 1989.

[21] A. Ouaissi, A. C. da Silva, A. G. Guevara, M. Borges, and E. Guilvard, "Trypanosoma cruzi-induced host immune system dysfunction: a rationale for parasite immunosuppressive factor(s) encoding gene targeting," Journal of Biomedicine and Biotechnology, vol. 1, no. 1, pp. 11-17, 2001.

[22] A. M. Acosta and C. A. Santos-Buch, "Autoimmune myocarditis induced by Trypanosoma cruzi," Circulation, vol. 71, no. 6, pp. 1255-1261, 1985.

[23] F. Kierszenbaum, "Chagas' disease and the autoimmunity hypothesis," Clinical Microbiology Reviews, vol. 12, no. 2, pp. 210-223, 1999.

[24] D. M. Engman and J. S. Leon, "Pathogenesis of Chagas heart disease: role of autoimmunity," Acta Tropica, vol. 81, no. 2, pp. 123-132, 2002.

[25] L. Klein, M. Hinterberger, G. Wirnsberger, and B. Kyewski, "Antigen presentation in the thymus for positive selection and central tolerance induction," Nature Reviews Immunology, vol. 9, no. 12, pp. 833-844, 2009.

[26] A. Basten and P. A. Silveira, "B-cell tolerance: mechanisms and implications," Current Opinion in Immunology, vol. 22, no. 5, pp. 566-574, 2010

[27] K. Pieper, B. Grimbacher, and H. Eibel, "B-cell biology and development," Journal of Allergy and Clinical Immunology, vol. 131, no. 4, pp. 959-971, 2013.

[28] A. A. Freitas and B. Rocha, "Population biology of lymphocytes: the flight for survival," Annual Review of Immunology, vol. 18, pp. 83-111, 2000.

[29] R. A. Willis, J. W. Kappler, and P. C. Marrack, "CD8 T cell competition for dendritic cells in vivo is an early event in activation," Proceedings of the National Academy of Sciences of the United States of America, vol. 103, no. 32, pp. 12063-12068, 2006.

[30] C. Montaudouin, M. Anson, Y. Hao et al., "Quorum sensing contributes to activated IgM-secreting B cell homeostasis," Journal of Immunology, vol. 190, no. 1, pp. 106-114, 2013.

[31] F. Y. Liew, M. T. Scott, D. S. Liu, and S. L. Croft, "Suppressive substance produced by T cells from mice chronically infected with Trypanosoma cruzi. I. Preferential inhibition of the induction of delayed-type hypersensitivity," Journal of Immunology, vol. 139, no. 7, pp. 2452-2457, 1987.

[32] F. Kierszenbaum, J. L. de Diego, M. Fresno, and M. B. Sztein, "Inhibitory effects of the Trypanosoma cruzi membrane glycoprotein AGC10 on the expression of IL-2 receptor chains and secretion of cytokines by subpopulations of activated human 
T lymphocytes," European Journal of Immunology, vol. 29, pp. 1684-1691, 1999.

[33] F. Kierszenbaum, M. Fresno, and M. B. Sztein, "The Trypanosoma cruzi membrane glycoprotein AGC10 inhibits human lymphocyte activation by a mechanism preceding translation of both, interleukin-2 and its high-affinity receptor subunits," Molecular and Biochemical Parasitology, vol. 125, no. 1-2, pp. 91101, 2002.

[34] L. van Overtvelt, M. Andrieu, V. Verhasselt et al., "Trypanosoma cruzi down-regulates lipopolysaccharide-induced MHC class I on human dendritic cells and impairs antigen presentation to specific CD8 ${ }^{+} \mathrm{T}$ lymphocytes," International Immunology, vol. 14, no. 10, pp. 1135-1144, 2002.

[35] S. Zambrano-Villa, D. Rosales-Borjas, J. C. Carrero, and L. Ortiz-Ortiz, "How protozoan parasites evade the immune response," Trends in Parasitology, vol. 18, no. 6, pp. 272-278, 2002.

[36] P. Alcaide and M. Fresno, "The Trypanosoma cruzi membrane mucin AgC10 inhibits T cell activation and IL-2 transcription through L-selectin," International Immunology, vol. 16, no. 10, pp. 1365-1375, 2004.

[37] F. R. Gutierrez, P. M. Guedes, R. T. Gazzinelli, and J. S. Silva, "The role of parasite persistence in pathogenesis of Chagas heart disease," Parasite Immunology, vol. 31, no. 11, pp. 673-685, 2009.

[38] A. M. Padilla, L. J. Simpson, and R. L. Tarleton, "Insufficient TLR activation contributes to the slow development of $\mathrm{CD}^{+} \mathrm{T}$ cell responses in Trypanosoma cruzi infection," Journal of Immunology, vol. 183, no. 2, pp. 1245-1252, 2009.

[39] M. J. Pinazo, G. Espinosa, C. Cortes-Lletget et al., "Immunosuppression and Chagas disease: a management challenge," PLOS Neglected Tropical Diseases, vol. 7, no. 1, Article ID e1965, 2013.

[40] A. Morrot, E. Terra-Granado, A. R. Pérez et al., "Chagasic thymic atrophy does not affect negative selection but results in the export of activated $\mathrm{CD}^{+} \mathrm{CD}^{+} \mathrm{T}$ cells in severe forms of human disease," PLoS Neglected Tropical Diseases, vol. 5, no. 8, Article ID e1268, 2011.

[41] V. Cotta-de-Almeida, Á. L. Bertho, D. M. S. Villa-Verde, and W. Savino, "Phenotypic and functional alterations of thymic nurse cells following acute Trypanosoma cruzi infection," Clinical Immunology and Immunopathology, vol. 82, no. 2, pp. 125-132, 1997.

[42] V. Cotta-de-Almeida, A. Bonomo, D. A. Mendes-da-Cruz et al., "Trypanosoma cruzi infection modulates intrathymic contents of extracellular matrix ligands and receptors and alters thymocyte migration," European Journal of Immunology, vol. 33, no. 9, pp. 2439-2448, 2003.

[43] W. Savino, D. A. Mendes-da-Cruz, S. Smaniotto, E. SilvaMonteiro, and D. M. Serra Villa-Verde, "Molecular mechanisms governing thymocyte migration: combined role of chemokines and extracellular matrix," Journal of Leukocyte Biology, vol. 75, no. 6, pp. 951-961, 2004.

[44] D. A. Mendes-da-Cruz, J. S. Silva, V. Cotta-de-Almeida, and W. Savino, "Altered thymocyte migration during experimental acute Trypanosoma cruzi infection: combined role of fibronectin and the chemokines CXCL12 and CCL4," European Journal of Immunology, vol. 36, no. 6, pp. 1486-1493, 2006.

[45] J. de Meis, A. Morrot, D. A. Farias-de-Oliveira, D. M. S. VillaVerde, and W. Savino, "Differential regional immune response in Chagas disease," PLoS Neglected Tropical Diseases, vol. 3, no. 7, article e417, 2009.

[46] M. Hofmann, A. Oschowitzer, S. R. Kurzhals, C. C. Krüger, and H. Pircher, "Thymus-resident memory $\mathrm{CD}^{+} \mathrm{T}$ cells mediate local immunity," European Journal of Immunology, vol. 43, no. 9, pp. 2295-2304, 2013.

[47] E. V. Acosta Rodriguez, E. I. Zuniga, C. L. Montes et al., "Trypanosoma cruzi infection beats the B-cell compartment favouring parasite establishment: can we strike first?" Scandinavian Journal of Immunology, vol. 66, no. 2-3, pp. 137-142, 2007.

[48] G. A. DosReis, "Evasion of immune responses by Trypanosoma cruzi, the etiological agent of Chagas disease," Brazilian Journal of Medical and Biological Research, vol. 44, no. 2, pp. 84-90, 2011.

[49] M. E. Giorgi and R. M. de Lederkremer, "Trans-sialidase and mucins of Trypanosoma cruzi: an important interplay for the parasite," Carbohydrate Research, vol. 346, no. 12, pp. 1389-1393, 2011.

[50] L. M. de Pablos and A. Osuna, "Multigene families in Trypanosoma cruzi and their role in infectivity," Infection and Immunity, vol. 80, no. 7, pp. 2258-2264, 2012.

[51] G. A. DosReis, C. G. Freire-de-Lima, M. P. Nunes, and M. F. Lopes, "The importance of aberrant T-cell responses in Chagas disease," Trends in Parasitology, vol. 21, no. 5, pp. 237-243, 2005.

[52] F. R. Gutierrez, P. M. Guedes, R. T. Gazzinelli, and J. S. Silva, "The role of parasite persistence in pathogenesis of Chagas heart disease," Parasite Immunology, vol. 31, no. 11, pp. 673-685, 2009.

[53] F. Nagajyothi, F. S. Machado, B. A. Burleigh et al., "Mechanisms of Trypanosoma cruzi persistence in Chagas disease," Cellular Microbiology, vol. 14, no. 5, pp. 634-643, 2012.

[54] I. A. Abrahamsohn and R. L. Coffman, "Cytokine and nitric oxide regulation of the immunosuppression in Trypanosoma cruzi infection," Journal of Immunology, vol. 155, no. 8, pp. 39553963, 1995.

[55] G. A. Martins, M. A. G. Cardoso, J. C. S. Aliberti, and J. S. Silva, "Nitric oxide-induced apoptotic cell death in the acute phase of Trypanosoma cruzi infection in mice," Immunology Letters, vol. 63, no. 2, pp. 113-120, 1998.

[56] M. P. Nunes, R. M. Andrade, M. F. Lopes, and G. A. DosReis, "Activation-induced $\mathrm{T}$ cell death exacerbates Trypanosoma cruzi replication in macrophages cocultured with $\mathrm{CD} 4^{+} \mathrm{T}$ lymphocytes from infected hosts," Journal of Immunology, vol. 160, no. 3, pp. 1313-1319, 1998.

[57] S. Schenkman, M. S. Jiang, G. W. Hart, and V. Nussenzweig, "A novel cell surface trans-sialidase of Trypanosoma cruzi generates a stage-specific epitope required for invasion of mammalian cells," Cell, vol. 65, no. 7, pp. 1117-1125, 1991.

[58] S. Schenkman, D. Eichinger, M. E. A. Pereira, and V. Nussenzweig, "Structural and functional properties of Trypanosoma trans-sialidase," Annual Review of Microbiology, vol. 48, pp. 499-523, 1994.

[59] J. O. Previato, C. Jones, M. T. Xavier et al., "Structural characterization of the major glycosylphosphatidylinositol membrane-anchored glycoprotein from epimastigote forms of Trypanosoma cruzi Y-strain," Journal of Biological Chemistry, vol. 270, no. 13, pp. 7241-7250, 1995.

[60] M. E. Giorgi and R. M. de Lederkremer, "Trans-sialidase and mucins of Trypanosoma cruzi: an important interplay for the parasite," Carbohydrate Research, vol. 346, no. 12, pp. 1389-1393, 2011.

[61] J. M. di Noia, D. O. Sanchez, and A. C. C. Frasch, "The protozoan Trypanosoma cruzi has a family of genes resembling the mucin genes of mammalian cells," Journal of Biological Chemistry, vol. 270, no. 41, pp. 24146-24149, 1995.

[62] C. A. Buscaglia, V. A. Campo, A. C. C. Frasch, and J. M. di Noia, "Trypanosoma cruzi surface mucins: host-dependent coat 
diversity," Nature Reviews Microbiology, vol. 4, no. 3, pp. 229236, 2006.

[63] L. Mendonça-Previato, L. Penha, T. C. Garcez, C. Jones, and J. O. Previato, "Addition of $\alpha$-O-GlcNAc to threonine residues define the post-translational modification of mucin-like molecules in Trypanosoma cruzi," Glycoconjugate Journal, vol. 30, no. 7, pp. 659-666, 2013.

[64] K. Joiner, A. Sher, T. Gaither, and C. Hammer, "Evasion of alternative complement pathway by Trypanosoma cruzi results from inefficient binding of factor B," Proceedings of the National Academy of Sciences of the United States of America, vol. 83, no. 17, pp. 6593-6597, 1986.

[65] R. T. Gazzinelli, M. E. S. Pereira, A. Romanha, G. Gazzinelli, and Z. Brener, "Direct lysis of Trypanosoma cruzi: a novel effector mechanism of protection mediated by human anti-gal antibodies," Parasite Immunology, vol. 13, no. 4, pp. 345-356, 1991.

[66] T. L. Kipnis, J. R. David, and C. A. Alper, "Enzymatic treatment transforms trypomastigotes of Trypanosoma cruzi into activators of alternative complement pathway and potentiates their uptake by macrophages," Proceedings of the National Academy of Sciences of the United States of America, vol. 78, no. 1, pp. 602605, 1981.

[67] V. L. Pereira-Chioccola, A. Acosta-Serrano, I. C. de Almeida et al., "Mucin-like molecules form a negatively charged coat that protects Trypanosoma cruzi trypomastigotes from killing by human anti- $\alpha$-galactosyl antibodies," Journal of Cell Science, vol. 113, no. 7, pp. 1299-1307, 2000.

[68] H. Erdmann, C. Steeg, F. Koch-Nolte, B. Fleischer, and T. Jacobs, "Sialylated ligands on pathogenic Trypanosoma cruzi interact with Siglec-E (sialic acid-binding Ig-like lectin-E)," Cellular Microbiology, vol. 11, no. 11, pp. 1600-1611, 2009.

[69] M. P. Nunes, B. Fortes, J. L. Silva-Filho et al., "Inhibitory effects of Trypanosoma cruzi sialoglycoproteins on $\mathrm{CD}^{+} \mathrm{T}$ Cells are associated with increased susceptibility to infection," PLoS ONE, vol. 8, no. 10, Article ID e77568, 2013.

[70] S. Pillai, I. A. Netravali, A. Cariappa, and H. Mattoo, "Siglecs and immune regulation," Annual Review of Immunology, vol. 30, pp. 357-392, 2012.

[71] D. A. Bermejo, S. W. Jackson, M. Gorosito-Serran et al., "Trypanosoma cruzi trans-sialidase initiates a program independent of the transcription factors ROR $\gamma \mathrm{t}$ and Ahr that leads to IL-17 production by activated B cells," Nature Immunology, vol. 14, pp. 514-522, 2013.

[72] H. Erdmann, C. Steeg, F. Koch-Nolte, B. Fleischer, and T. Jacobs, "Sialylated ligands on pathogenic Trypanosoma cruzi interact with Siglec-E (sialic acid-binding Ig-like lectin-E)," Cellular Microbiology, vol. 11, no. 11, pp. 1600-1611, 2009.

[73] T. Jacobs, H. Erdmann, and B. Fleischer, "Molecular interaction of Siglecs (sialic acid-binding Ig-like lectins) with sialylated ligands on Trypanosoma cruzi," European Journal of Cell Biology, vol. 89, no. 1, pp. 113-116, 2010.

[74] P. R. Crocker, J. C. Paulson, and A. Varki, "Siglecs and their roles in the immune system," Nature Reviews Immunology, vol. 7, no. 4, pp. 255-266, 2007.

[75] Z. Yu, M. Maoui, L. Wu, D. Banville, and S. Shen, "mSiglec-E, a novel mouse CD33-related siglec (sialic acid-binding immunoglobulin-like lectin) that recruits Src homology 2 (SH2)domain-containing protein tyrosine phosphatases SHP-1 and SHP-2," Biochemical Journal, vol. 353, no. 3, pp. 483-492, 2001.

[76] J. Q. Zhang, B. Biedermann, L. Nitschke, and P. R. Crocker, "The murine inhibitory receptor $\mathrm{mSiglec}-\mathrm{E}$ is expressed broadly on cells of the innate immune system whereas mSiglec-F is restricted to eosinophils," European Journal of Immunology, vol. 34, no. 4, pp. 1175-1184, 2004.

[77] T. L. Tinder, D. B. Subramani, G. D. Basu et al., "MUC1 enhances tumor progression and contributes toward immunosuppression in a mouse model of spontaneous pancreatic adenocarcinoma," Journal of Immunology, vol. 181, no. 5, pp. 3116-3125, 2008.

[78] P. Allavena, M. Chieppa, G. Bianchi et al., "Engagement of the mannose receptor by tumoral mucins activates an immune suppressive phenotype in human tumor-associated macrophages," Clinical and Developmental Immunology, vol. 2010, Article ID 547179, 10 pages, 2010.

[79] M. Ohta, A. Ishida, M. Toda et al., "Immunomodulation of monocyte-derived dendritic cells through ligation of tumorproduced mucins to Siglec-9," Biochemical and Biophysical Research Communications, vol. 402, no. 4, pp. 663-669, 2010.

[80] T. Freire, R. Lo-Man, S. Bay, and C. Leclerc, "Tn glycosylation of the MUC6 protein modulates its immunogenicity and promotes the induction of Th17-biased T cell responses," Journal of Biological Chemistry, vol. 286, no. 10, pp. 7797-7811, 2011.

[81] A. Anandkumar and H. Devaraj, “Tumor Immunomodulation: mucins in resistance to initiation and maturation of immune response against tumors," Scandinavian Journal of Immunology, vol. 78, no. 1, pp. 1-7, 2013.

[82] C. Charvet, A. J. Canonigo, S. Bécart et al., "Vavl promotes T cell cycle progression by linking TCR/CD28 costimulation to FOXO1 and p27kip1 expression," Journal of Immunology, vol. 177, pp. 5024-5031, 2006.

[83] A. Jatzek, M. M. Tejera, A. Singh, J. A. Sullivan, E. H. Plisch, and M. Suresh, "p27(Kip1) negatively regulates the magnitude and persistence of CD4 T cell memory," Journal of Immunology, vol. 189, pp. 5119-5128, 2012.

[84] C. M. Sawai, J. Freund, P. Oh et al., "Therapeutic targeting of the cyclin D3:CDK4/ 6 complex in T cell leukemia," Cancer Cell, vol. 22, pp. 452-465, 2012.

[85] A. Deshpande, P. Sicinski, and P. W. Hinds, "Cyclins and cdks in development and cancer: a perspective," Oncogene, vol. 24, no. 17, pp. 2909-2915, 2005.

[86] W. Wu, D. C. Munday, G. Howell, G. Platt, J. N. Barr, and J. A. Hiscox, "Characterization of the interaction between human respiratory syncytial virus and the cell cycle in continuous cell culture and primary human airway epithelial cells," Journal of Virology, vol. 85, no. 19, pp. 10300-10309, 2011. 


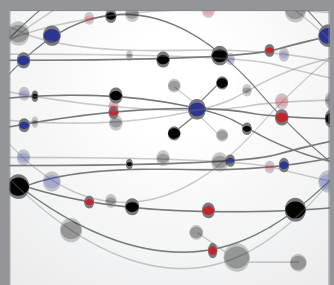

The Scientific World Journal
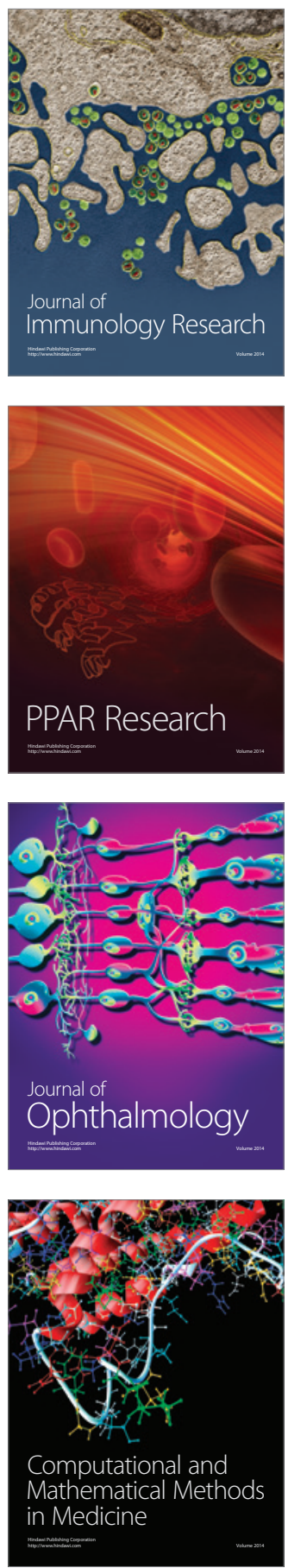

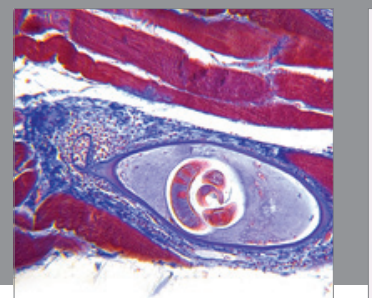

Gastroenterology

Research and Practice
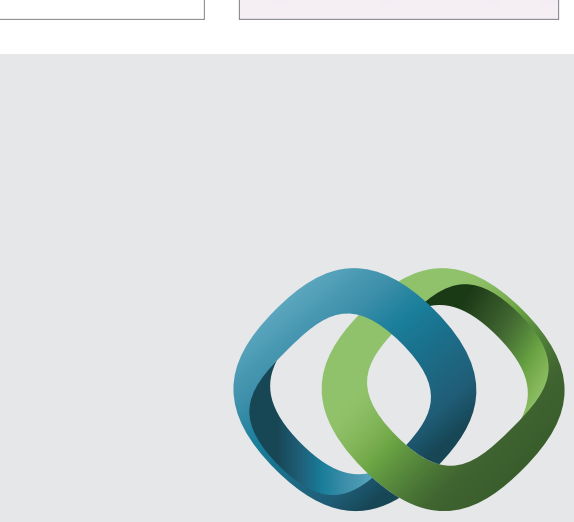

\section{Hindawi}

Submit your manuscripts at

http://www.hindawi.com
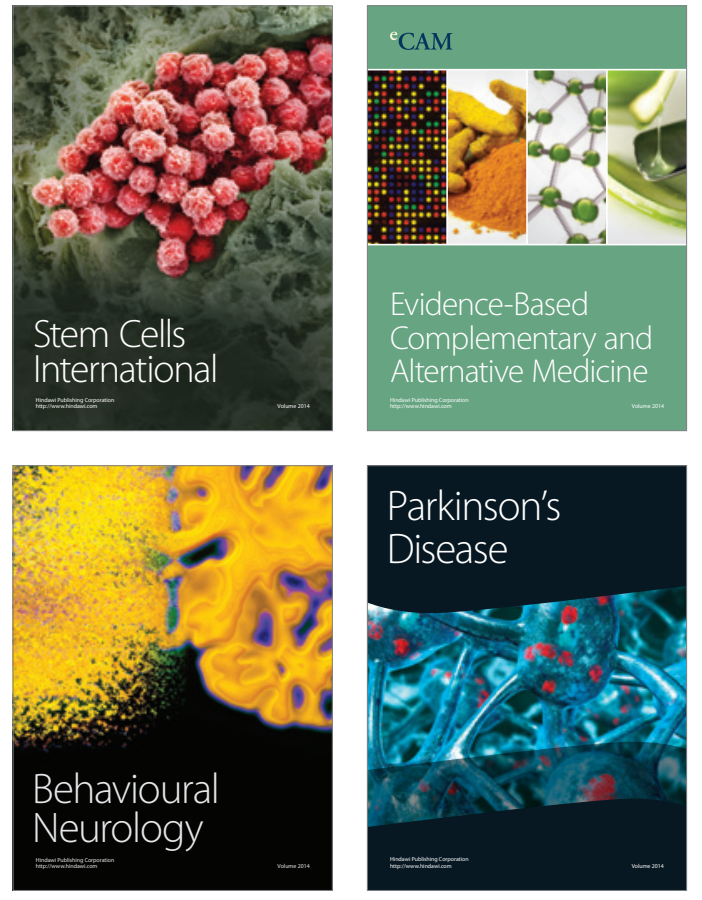
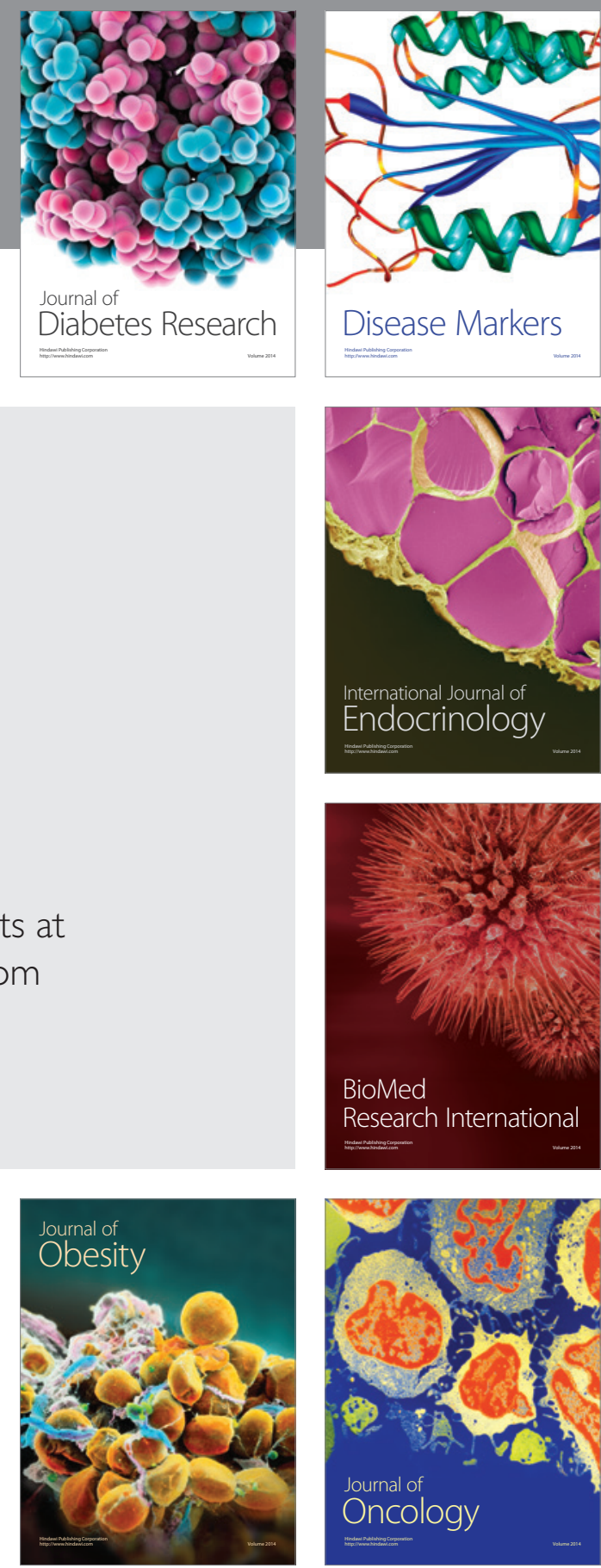

Disease Markers
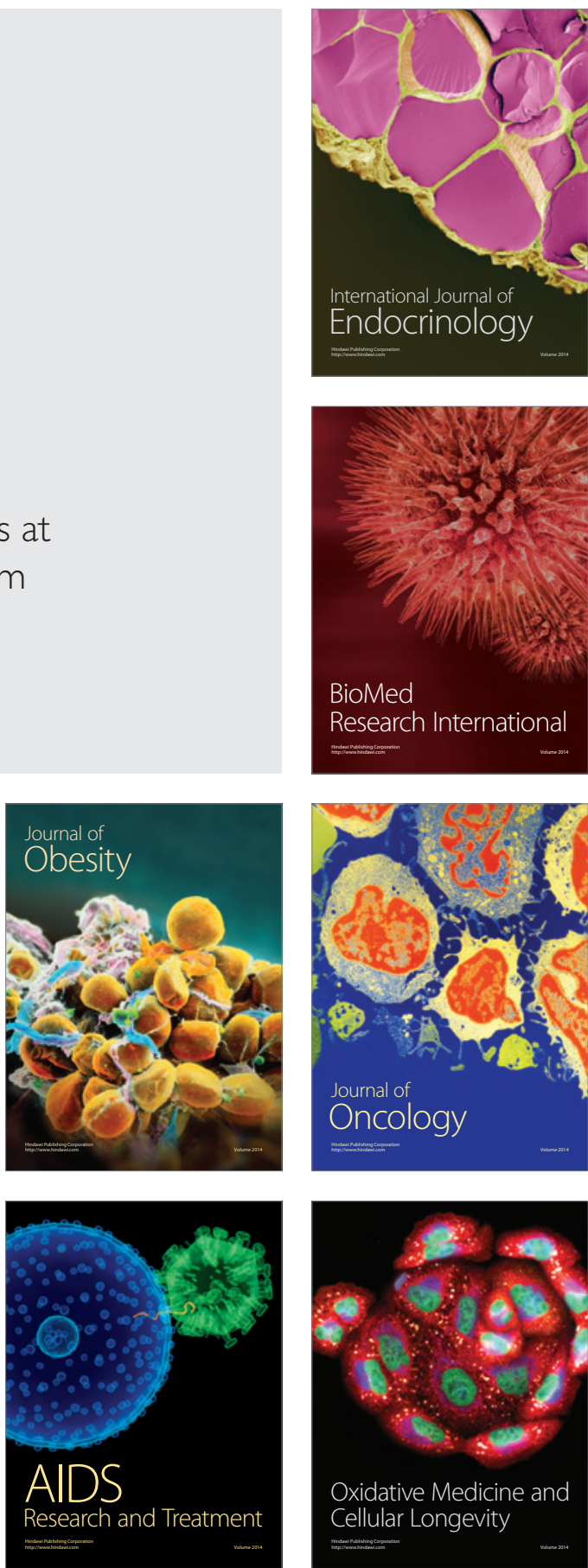